\title{
The X-10: A Revolution in Knee Rehabilitation
}

\author{
David K. Halley, $M D^{\dagger}$, Paul Ewing, BME, $M B A^{*}$
}

\section{Abstract:}

The $\mathrm{X}-10$ is an innovative knee rehabilitation machine that uses Variable Pressure technique to move the knee below the patient's pain threshold, within days rather than weeks, focusing on terminal flexion and extension. Pumping fluid away from the knee during the first two stages of fibrosis prevents the ultimate formation of scar tissue.

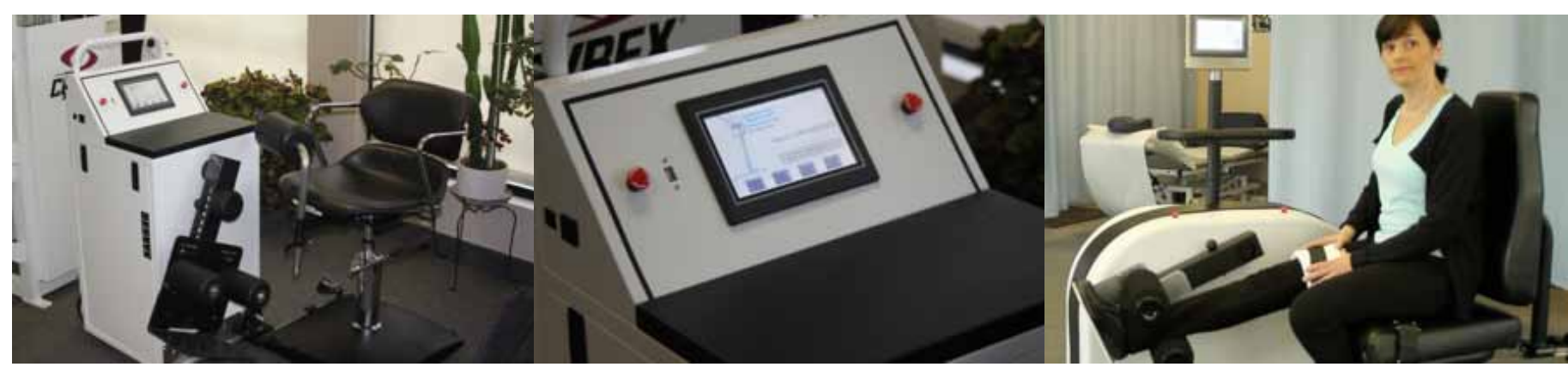

More than 500,000 patients underwent total knee arthroplasty (TKA) in 2012 in the United States alone, a number that is expected to exceed $3,480,000$ by the year $2030 .{ }^{14}$ The rehabilitation process for TKA patients is extensive, costly, and does not always yield optimal results. Many patients struggle to regain full mobility following TKA because stiffness in the knee joint can quickly progress to scar tissue in a short period of time. If this process is not prevented, scar tissue may impede flexibility in the future. Lack of full range of motion not only affects gait and mobility, but can also lead to future back and hip pain. It is well recognized that rapidly restoring range of motion and rebuilding muscle strength is critical following any type of knee procedure. . $22,27,37^{2}$

Von Riemke, in his presidential address to the Danish Surgical Society stated in 1926 that "all joint affections... should be moved and movement should begin on the first day, should be slow, and as much as possible, it should be continuous". ${ }^{26}$
Based upon his experimental investigation on rabbit knees in the early 1960 's, Salter ${ }^{26,27,34,35}$ came to the conclusion that immobilization is unhealthy for a joint. He was of the opinion that intermittent motion was healthier for a knee joint as compared to immobilization, and he arrived at the conclusion that perhaps continuous motion might be better. He was quick to realize that skeletal muscle had easy fatigability so that if one were to have continuous motion, it would have to be passive rather than active.

There are several ways to describe continuous passive motion but one definition can be, "a machine with an external motor which passively moves a joint through a pre-set arc of motion. ${ }^{17}$

\footnotetext{
+ Co-Founder \& Medical Director

* Co-Founder, President \& CEO

Halley Orthopedics

Columbus, Ohio

www.x10therapy.com
} 
This article has been written to describe a newly designed rehabilitation machine, called the "X-10". The $\mathrm{X}-10$ works in a radically different manner from the previous 'traditional' continuous passive motion machines used in rehabilitation of the knee.

O'Driscoll has pointed out that early research on continuous passive motion was based upon the theory that it promoted healing of articular cartilage. ${ }^{26,34,35}$ However, throughout the mid 1980's and 1990's, the major use of traditional continuous passive motion has been to prevent stiffness following total knee arthroplasty.

In the 1990's there was an explosion in the use of continuous passive motion with many early advocates supporting such treatment. . $7,11,12,16,18,21,22,29,32,36,37$ However, with time and appropriate evaluation of results, a significant group of detractors stated CPM was of no benefit in the rehabilitation of total knee arthroplasty because results were the same at six months to one year following surgery, whether or not CPM was used. ${ }^{3,5}$ $, 6,8,9,10,14,17,18,19,20,21,24,25,29,31,32,33,36$

O'Driscoll ${ }^{26}$ suggested perhaps it mattered how CPM was used and to better understand the potential for continuous passive motion one must understand the pathophysiology. It is important to fully understand the theory behind the development of fibrosis before interpreting the past experience with continuous passive motion.

\section{Pathophysiology of Joint Stiffness}

The pathophysiology of joint stiffness, as theorized by O'Driscoll ${ }^{25,26}$, describes the four stages of fibrosis with progression of the first three stages leading into the fourth stage of fibrosis characterized by thick hard dense scar formation.

Stage one: Bleeding into the joint is the first stage of fibrosis. This occurs in a matter of minutes to hours following knee surgery, resulting in the distention of the joint capsule and periarticular swelling. The maximum capsular capacity of the human knee joint occurs at 35 degrees of flexion. This swelling leads to very high intraarticular hydrostatic pressure and any movement from the maximum capacity of the joint increases these pressures which cause severe pain. The patient attempts to hold the knee in the position of maximum capacity so as to minimize the pain created by any increase in pressure. This selfprotective mechanism to pain contributes to the early loss of motion in flexion and extension.

Stage Two: The second stage is edema which occurs in a matter of hours or days. The edema is caused by mediators released from platelets as well as injured or dead cells. These cause nearby blood vessels to dilate and leak plasma resulting in swelling of the periarticular tissues, thereby decreasing the compliance of this tissue making it more difficult to move the joint.

The effects of these first two stages are a result of fluid accumulation. This is why it is so important to start treatment early to pump the fluid from the knee and periarticular tissues as soon as possible. In the next two stages it is significant to note this fluid is replaced by an extracellular matrix.

Stage Three: The third stage occurs in a few days to weeks with the formation of granulation tissue which has the physical properties between that of a well-formed blood clot and loose areolar fibrous tissue. That stiffness, originally caused by fluid accumulation, is now due to the deposition of a solid extracellular matrix.

Stage Four: As the collagen hardens it becomes more and more difficult to eliminate. This progression typically finalizes into the fourth stage of fibrosis creating dense hard scar tissue which can permanently impede mobility within two to four weeks, when many patients are just beginning physical therapy.

O'Driscoll et $\mathrm{al}^{25}$ have shown that flexion and extension of the knee create changes in pressure in a sinusoidal fashion which results in a "pumping effect" which is responsible for clearing blood and edema from the joint.

When patient physical therapy typically begins, lack of range of motion is not normally a focus point during the first few weeks after surgery. The knee is swollen, stiff, painful and the patient is simply trying to walk short distances. By the time outpatient physical therapy begins (on average 3-4 weeks post-TKA) it is often not possible to prevent the accumulation of fluid in the periarticular tissue. Failure to achieve a full range of motion in the immediate postoperative period, combined with 
permitting the accumulation of even relatively small amounts of periarticular blood and edema, permits collagenous scar tissue formation. Full range of motion might never be completely achieved.

A device and method for early removal of fluid from the periarticuar tissue prior to collagen formation would therefore be desirable. Treatment should begin early, working in the terminal range of flexion and extension, and continued until all swelling of the knee has been eliminated.

\section{The X-10: New Rehabilitation Technology}

The $\mathrm{X}-10$ is a new technology that will allow continuous passive motion to work as originally desired, but never accomplished with the traditional CPM machines.

The traditional continuous passive motion (CPM) machine undesirably sets limits on extension and flexion and operates only within those limits. If the limits are set too aggressively, the joint can experience excess stress, leading to pain and potential injury, while too little pressure results in insufficient progress. Typically, the traditional CPM machines are used to exercise a specified range of motion limited by fixing the target angles within the patient's existing range of motion, which is already achievable by the patient.

It is best to work in the terminal range of flexion and extension as this is more effective in pumping the fluid from the joint. ${ }^{25,26}$ Working in the midrange of motion, already achieved by the patient, becomes self-limiting and can undesirably leave periarticular fluid about the joint and prevent meaningful progress. In fact, it can be detrimental as this retained fluid can ultimately lead to undesired scar formation.

Traditional continuous passive machines (CPM) depend upon pre-programmed flexion and extension values to determine the extent of motion. These machines will push blindly and have no pressure feed-back and no pressure variability.

The traditional CPM machine is unable to provide a high or low amplitude 'pause' at the extremes of the patient's range of motion. The X-10 slows down and literally coasts into the last five degrees of terminal flexion or extension, at which time it stops for a programmed number of seconds. This allows the tissue to maintain a 'stretch' for the individual's programmed time of stretch and relaxation.

Another issue is that not all patients respond in the same manner to their therapy. Some patients tend to form scar tissue more rapidly and this hypertrophic scar formation leads to loss of function at a faster pace than normal.

Orthopedic surgeons have always been leaders in the development and use of new technology in the treatment and care of their patients. It is our goal to continue this tradition into the field of joint rehabilitation offering a technological therapy aid to help the surgeon or physical therapist provide better care to their patients.

Understanding the basic pathophysiology of fibrosis and the importance of early use of continuous passive motion, brings us to the problem associated with the use of traditional CPM machines. . .Pain! Pain prevented patients from starting early and working in terminal range of extension and flexion.

The X-10 works in a radically different way than a traditional CPM machine. Rather than having the knee move back and forth between two fixed points at a constant pressure (like the traditional CPM machine), the X-10 uses the newly patented technology of Variable Pressure. It uses threshold pressure to operate, allowing the knee angle to be variable. This customizable "pressure threshold" is set by identifying the maximum pressure tolerable within the patient's comfort zone. The primary focus is on the patient's current terminal range of extension and flexion. Large arcs of motion, working at terminal limits, greatly enhances the pumping action eliminating blood and periarticular fluid while gently stretching and re-aligning the muscle fibers.

\section{How Does The X-10 Control Pain?}

By limiting pressure, pain is taken out of the equation. The "Variable Pressure" technology allows for customizable "Pressure Threshold" to be set by identifying the maximum pressure tolerable within the patient's comfort zone. Never will the pressure exceed this 'ceiling' pressure which protects the patient and provides assurance to the 
patient that there will be no sudden increased pain experience throughout the entire rehabilitation process. As the patient quickly becomes accustomed to the pressure limit, additional pressure can be added in one-pound increments as tolerated by the patient. This increase in pressure increases further the current range of motion attained.

The idea behind the X-10 is the patient can work at a pressure slightly less than a level that will cause pain. After several cycles of the machine the patient will plateau in motion levels as the patient has quickly adjusted to that particular pressure thereby allowing the therapist to increase the pressure, as needed, in one-pound increments. These incremental increases in pressure allow for further progression of motion. Thus, an increase in pressure increases motion. That motion plateaus. Next, the pressure is increased in one-pound increments, as tolerated by the patient, which increases motion that will plateau. This process is repeated over and over until the final desired range of motion is achieved. This process is very gentle and creates very little discomfort for the patient.

\section{The X-10 Has Four Flexibility Programs}

There is a "warm-up" program which is very popular with patients. It functions at any range of motion short of terminal flexion and extension. Once warmed up the patient can proceed in working at terminal limits which is the second program. In this program the machine makes a full cycle between flexion and extension at terminal limits. The third and fourth programs have to do with specifically working at either end of the spectrum in flexion or extension thereby short-cutting a full cycle. This was designed to save valuable rehabilitation time. For example, if the patient has full extension, there is no need to work on extension. The machine can be programmed in a short cycle working only in terminal degrees of flexion. Vice-versa, if good flexion has been achieved and there is lack of full extension, the machine is programmed to work the short cycle in terminal extension.

The ability to prevent flexion contractures is a result of the two modes of using terminal extension with the $\mathrm{X}-10$. The $\mathrm{X}-10$ was originally designed to simply prevent flexion contractures: thus the name reflects ' $\mathrm{X}-10$ ' for 'extend', to prevent flexion contractures.

\section{Strengthening}

The $\mathrm{X}-10$ has two programs to allow for eccentric and concentric strengthening of the thigh to be used either before or after surgery. Before surgery, a baseline record of strength can be identified. From this point a patient can proceed with strengthening programs prior to surgery. This can prevent any further loss of strength resulting from the basic disease process or from the normal decrease in strength following any surgical procedure.

"Quadriceps strength ${ }^{27}$ was the strongest predictor at one year...(and) functional decline may be delayed with adequate quadriceps training. Followup of these patients continues to discern the longterm impact of strengthening interventions". When using strengthening programs after surgery, we recommend waiting three weeks. Though the machine is gentle and can work in one-pound increments of pressure, we do not want to risk injury to the capsular repair until sufficient healing has occurred.

\section{Patient Safety}

Patient safety has always been top priority in the development of the X-10. There are five built-in safety mechanisms, including eStop, that allows the user to stop the motor and treatment immediately.

\section{X-10 Is Not Cookie Cutter Therapy!}

We recognize that patients respond differently to therapy for a variety of reasons.

One of the highlights of this machine is its ability to allow patient therapy to be individualized.

Beginning early treatment allows the healthcare provider to identify any potential problem that will require focusing upon special treatment.

\section{Additional Benefits}

The X-10 incorporates a visual computer module which collects information while the patient is on the machine, recording initial and final range of 
motion for each session, time of use, and pressures used for treatment. This information can be printed and used for billing purposes, saving physical therapists considerable time in documentation, or it can be electronically sent to physicians involved in the care of the patient. Lastly, the computer module provides real-time positional location of the leg,visualized on the computer screen, allowing patients to focus on their rehabilitation at the very instant of treatment, acting as a "coaching" aid for them during their treatment.

\section{Comfort}

The X-10 answers two of the criticisms of the traditional CPM machine. The first is that the patient lies in bed in a supine position. ${ }^{1}$ With the X-10 the patient is comfortably seated in an attached specially designed chair which can swivel into a locked position to allow treatment of the right or left knee. The chair is so designed as to relieve pressure from the hamstring muscles and sciatic nerve to avoid any contribution to the development of back pain. The second criticism ${ }^{3}$ of the traditional CPM machine is the recorded motion in extension and flexion is usually less than the "true" range of motion. With the X-10, the exact position of the leg in space is computer generated, based upon the position of the arm of the mechanical leg holder, and is accurate within 1-2 degrees.

\section{CPM Case Studies}

The X-10, having been recently developed, has not allowed for large numbers of patients for study. However, there has been a tremendous positive response from all patients who have used the $\mathrm{X}-10$. We can report three classic patient scenarios, representative of conditions we, as surgeons, have to face in the care of our patients. Each patient scenario has been carefully studied.

The first scenario is a right knee that had advanced long term loss of flexion. Ritter et al state that patients with less than 75 degrees of flexion before surgery were less likely to improve postoperatively. ${ }^{5,29}$

A second scenario, a left knee (the opposite knee in the same patient) had a more normal pre-operative range of motion and should represent the course of the typical average patient knee.

The third case scenario involves a patient who had received regular physical therapy for three weeks, and plateaued, and was dissatisfied with her range of motion. This case started later in therapy with the X-10 and suggests benefit can be attained even when starting later than normal.

Case \# 1: A severely limited right knee joint with marked loss of flexion before surgery, present for 16 years, had a pre-op AGE 15 degrees and AGF of 70 degrees. The patient began using the X-10 at home 3 days after surgery; it was used 2-3 times / day for 15-20 minutes, followed by ice therapy. That knee reached full extension as well as 108 degrees of flexion within 3 weeks allowing the patient to go up and down stairs in reciprocal fashion and ride a stationary bicycle, two things she had not been able to do for 16 years.

Case \# 2: This is the same patient with a more 'typical' left knee (AGE 15 degrees and AGF 115 degrees), operated upon six weeks following her first knee surgery. Again, she started at home on post-op day 3, and within one week from surgery, was off all external support and had reached full extension and 116 degrees of flexion.

Case \#3: A middle aged female with goal of returning to golf, tennis, etc., began using the $\mathrm{X}-10$ in an outpatient clinic 3 weeks post surgery after experiencing disappointing results with routine physical therapy alone. At that time her AGE was 5 degrees and AGF 89 degrees. After 4 weeks of X-10 she had AGE of 0 degrees and AGF of 134 degrees having increased her total ROM by 50 degrees while using the X10.

\section{Conclusion}

It seems a reasonable assertion that if a patient can comfortably begin rehabilitation within two to three days following surgery (made possible because of Variable Pressure) there is reasonable expectation that patients will achieve a greater range of motion in a shorter period of time. However, this is a secondary goal. We are thinking in "revolutionary" terms. It is our primary goal that patients will have a more rapid recovery allowing them to become totally independent, and if a member of the work force, able to return to work in a shorter period of 
time following surgery. Our dream is that time frame will be four to six weeks rather than the traditional three months. If this becomes possible it will achieve considerable health care savings.

\section{References}

1. Beaupre L, Davies D, Jones C,Cinats J. Exercise combined with continuous passive motion or slider board therapy compared with exercise only: a randomized controlled trial of patients following total knee arthroplasty. Phys Ther 2001(81)1029-1037).

2. Bennett L, Brearley S, Hart J, Bailey M, A comparison of 2 continuous passive motion protocols after total knee arthroplasty: a controlled and randomized study. J Arthroplasty, 2005;20(2):225-33 (ISSN: 088305403).

3. Bible J, Simpson A, Debdut B, Pelker R, Grauer J Actual knee motion during continuous passive motion protocols is less than expected. Clin Orthop Relat Res 2009 October;4679100: 2656-2661.

4. Chen B, Zimmerman J, Soulen L, DeLisa J. Continuous passive motion after total knee arthroplasty: a prospective study. Am J Phys Med Rehab 2000; 79L421-426.

5. Chiarello C, Gunderson L, O'Halloran T. The affect of continuous passive motion duration and increment on range of motion in total knee arthroplasty patients. JOSPT 25(2) Feb 1997.

6. Colwell CS jr, Morris BA. The influence of continuous passive motion on the results of total knee arthroplasty. Clin Orthop 1992 (2760;225-8

7. Coutts RD, Toth C, Kaita JH. The role of continuous passive motion in the rehabilitation of the total knee patient. In: Jungerford DS, Krackow KA, Kenna RV, eds. Total knee arthroplasty: a comprehensive approach. Baltimore: Williams and Wilkins, 1984:126-32. INTERVIEW

8. Dennis M, Moffet H, Caron F, Ouellet D, IPaquet J, Nolet L. Effectiveness of continuous passive motion and conventional physical therapy after total knee arthroplasty: a randomized clinical trial. Physical Therapy Journal Feb 2006; 86 (2) 174-185

9. Dorr LD. Continuous passive motion offers no benefit to the patient Orthopedics .1999;22:393.

10. Harvey L, Brosseau H, Herbert R. Continuous passive motion following total knee arthroplasty in people with arthritis. Cochran Daabase Syst Rev. 2010 Mar 17;(3):CD004260.

11. Johnson $\mathrm{D}$, The effect of continuous passive motion on wound healing and join mobility after knee arthroplasty. J Bone Joint Surg Am. 1990 Mar; 72(3):421-6.

12. Jordan L, Siegel J, Olivo J. Clin Orthop Relat res. 1995 June;(315L231-3

13. Kumar PJ, McPharson EJ, Dorr LD, Wan Z, Baldwin K. Rehabilitation after total knee arthroplasty: a comparison of 2 rehabilitation techniques. Clin Orthop Relat Res. Oct. 1996; 331: 93-101.

14. Kurtz S, Ongk, Lau E, Mowat F, Halpern M. Projections of primary and revision hip and knee arthroplasty in the united states from 2005 to 2030. J Bone Joint Surg Am 2007, April, 89 (4): 780-785.

15. Lachiewicz $P$. The role of continuous passive motion after total knee arthroplasty: paper presented at the knee society meeting 2000. Clin Orth Rel Res. Nov 2000 (380) 144-150.

16. Lenssen A, Crijns Y, Waitje E, Roox G, van Steyn M, Geesink R, van den Brandt P, de Bie R, Design of prolonged use of continuous passive ( (CPM)as an adjuct to physiotherapy following total knee arthroplasty: design of a randomized control trial [ ISRCTN85759656]. 2006 $\mathrm{Feb}(23) ; 7: 15$.

17. Lenssen A, Van Steyn M, Crijns Y, Waltie E, Roox G, Geesink R, van den Brandt P, De Bie R. Effectiveness of prolonged use of continuous passive motion (CPM), as an adjunct to physiotherapy, after total knee arthroplasty. BMC Musculoskelet Disord, 2008: 9: 60.

18. MacDonald S, Bourne R, Rorabeck C, McCalden R, Kramer J, Vaz M. Prospective randomized clinical trial of continuous passive motion after total knee arthroplasty. Clin Orthop Rel Res 2000 Nov;(380):30-5.

19. Maloney WJ, Shurman DJ, Hangen D, Goodman SB, Edworthy S, Bloch DA. The influence of continuous passive motion on outcome in total knee arthroplasty. Clin Orthop 1990 (256):162-8.

20. McInnes J, Larso M, Daltroy L, Brown T, Fossel A, Eaton H, ShulmanKirwan B, Steindorf S, Poss R, Liang M. A controlled evaluation of continuous passive motion in patients undergoing total knee arthroplasty JAMA 1992;Sept 16;268(11): 1423-8.

21. Milne S, Brosseau L, Robinson V, Noel M, Davis J, Drouin H, Wells G, Tugwell P. Continuous passive motion following total knee arthroplasty. Update In Cocharane Databasep Syst Rev. 2010(3):CD004260.

22. Mizner R, Petterson S, Snyder-Mackler L. Quadriceps strength and the time course of functional recovery after total knee arthroplasty. $J$. Orthop Sports Phys Ther vol 35, number 7, July 2005L425-436.

23. Montgomery F, Eliason M. Continuous passive motion compared to active physical therapy after knee arthroplasty: similar hospitalization times in a randomized study of 68 patients Acta Orthop scand 1996:67 (1) 7 -9.

24. Nadler S, Malanna R, Zimmerman J. Continuous passive motion in the rehabilitation setting: a retrospective study. AM J Phys Med Rehabil. 1993; 72:162-165.

25. O'Driscoll SW, Kumar A, Salter RB. The effect of continuous passive motion on the clearance of a hemarthrosis from a synovial joint; and experimental investigation in the rabbit. Clin Orthop 1983; 176L30511 .

26. O"Driscoll SW, Giori NJ. Continuous passive motion (CPM): theory and principles of clinical application. J Rehabil Res Dev. Mar-Apr 2000;37:179-188.

27. Peterson S, Mizer R, Stevens J, Raisis L, Bodenstab A, Newcomb W, Snyder-Mackler L, Improved function from progressive strengthening interventions after total knee arthroplasty: a randomized clinical trial with an ibedded prospective cohort. Arthritis and Rheumatism Vol. 61, No. 2, February 15, 2000, pp 174-183.

28. Pope RO, Corcoran, S, McCaul K, Howie DW. Continuous passive motion after primary total knee arthroplasty. J Bone Joint Srug $\{\mathrm{Br}\}$ 1997;79-B:914-7

29. Ritter M, Stringer E. Predictive range of motion after total knee replacement. Clin Orthop 1979; 143: 115-9.

30. Ritter MA, Gandolf VS, Holston KS. Continuous passive motion versus physical therapy in total knee arthroplasty. Clin Orthop Relat Res. 1989 Jul;(244):239-43.

31. Romness D, Rand J. The role of continuous passive motion following total knee arthroplasty. Clin Orthop Relat Res, 1988 Jan: (226): 34-37.

32. Rorabeck $\mathrm{CH}$ continuous passive motion is a useful postoperative tool [see comments]. Orthopedics 1999;22(4):392.

33. Salter RB, Simmonds DF, Malcom BW, et al. The biological effect of continuous passive motion on the healing of full-thickness defects in articular, cartilage: an experimental investigation in the rabbit. $J$ Bone Joint Surg \{AM\} 1980;62-A:1232-51.

34. Salter RB. The biologic concept of continuous passive motion of synovial joints. The first 18 years of basiac research ad its clinical application. Clin Orthop Relat Res. May 19891242:12-25.

35. Sing Ki, Kwong Chu. Use of continuous passive motio after total knee arthroplasty. J Arthroplasty, April 2001; Vol 16 (3) 330-339.

36. Ververeli P, D, Hearn S, Booth R, Hozak W, Rothman R . Continuous passive motion after total knee arthroplasty. Analysis of cost and benefits. Clin Orthop Relat Res 1995 Dec;(321):208-15.

37. Young A, Current Issues in Arthrogenous inhibition, Annals of the Rheumatic Diseases 1993; 52: 829-834. 